\title{
Orbital Cavernous Hemangioma Presenting With Atypical Appearance
}

\author{
-Case Report-
}

\author{
Osamu AKIYAma, Satoshi TSUTSumi, Yasuo SugA, \\ Yusuke ABE, Yukimasa YASUMOTO, and Masanori ITO
}

Department of Neurological Surgery, Juntendo University Urayasu Hospital, Urayasu, Chiba

\begin{abstract}
A 25-year-old male presented with unilateral retroorbital dysesthesia persisting for 2 weeks followed by progressive palpebral ptosis without preceding trauma, paranasal sinus surgery, or infectious signs. Neuroophthalmological inspection revealed mildly increased intraocular pressure and exophthalmos on the affected side, without conjunctival chemosis, restriction of the extraocular movements, double vision, or visual impairment. These symptoms did not vary with posture, straining, and Valsalva maneuver. Neuroimaging showed an irregularly-shaped orbital mass without enhancement mainly situated in the medial compartment of the orbit and encasing the optic nerve. Bruit was not audible and abnormal vasculatures were not identified in and around the affected orbit. Transcranial surgical exploration through the surgical window formed by the superior and medial rectus muscles revealed a purplish elastic-soft mass, heterogeneous in consistency and tightly adhering to the surrounding tissue, so cryoprobe-assisted radical tumor resection could not be completed without jeopardizing the optic nerve. The mass was subtotally resected piecemeal without postoperative visual impairment. The histological diagnosis was cavernous hemangioma. Orbital cavernous hemangiomas may present with an atypical appearance which confuses pretreatment diagnosis and makes surgical extirpation more hazardous. Conservative surgery should be indicated for poorly demarcated orbital cavernous hemangiomas considering the usual benign clinical course and postoperative sequelae.
\end{abstract}

Key words: orbital cavernous hemangioma, orbital tumor, surgery

\section{Introduction}

Orbital cavernous hemangiomas $(\mathrm{CHs})$ are the commonest type of benign primary orbital tumor in adults, and account for $25 \%$ of intraconal orbital tumors in Japan. ${ }^{6,8,10)}$ Orbital CH has a typical macroscopic appearance of a dark plum-colored mass with variable trabeculated surface depending on the thickness of the fibrous capsule and septae. ${ }^{8)}$ Surgical resection is indicated in symptomatic patients presenting impaired visual function or ocular movements, progressing exophthalmos, and symptoms of increased intraorbital pressure. ${ }^{1,5,7,10)}$ Orbital $\mathrm{CH}$ has specific neuroimaging findings of circumscribed enhanced mass, which indicates that total resection is feasible without postoperative worsening of visual impairment. ${ }^{3,7,8)}$ However, some orbital $\mathrm{CHs}$ have had tight adhesion with the surrounding tissue without the circumferential capsule, which makes

Received July 1, 2008; Accepted December 26, 2008 surgical resection hazardous. ${ }^{1)}$ These atypical orbital $\mathrm{CHs}$ are currently thought to be a clinicopathologically distinct entity from the $\mathrm{CHs}$ originating in the central nervous system (CNS). ${ }^{3)}$

We describe a case of orbital $\mathrm{CH}$ with atypical appearance and clinicopathologic findings.

\section{Case Report}

A 25-year-old male complained of unilateral retroorbital dysesthesia persisting for 2 weeks followed by progressive palpebral ptosis. His past medical history was unremarkable for hypertension, hematological disorder, thyroid disease, sarcoidosis, and paranasal sinus surgery. He was unaware of either recent head injury or preceding infectious signs. He consulted a local ophthalmology clinic, and was referred to our hospital under a diagnosis of suspected orbital tumor.

Examination identified mildly increased intraocular pressure and exophthalmos, without conjunc- 


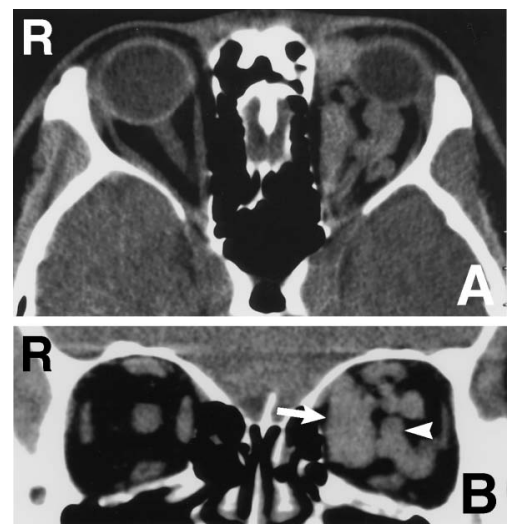

Fig. 1 Axial (A) and coronal (B) computed tomography scans demonstrating an irregularly-shaped intraconal mass mainly occupying the medial compartment of the orbit, appearing as homogeneously isodense, and poorly separated from the medial rectus muscle (arrow), without findings of intralesional calcification. Arrowhead: optic nerve.
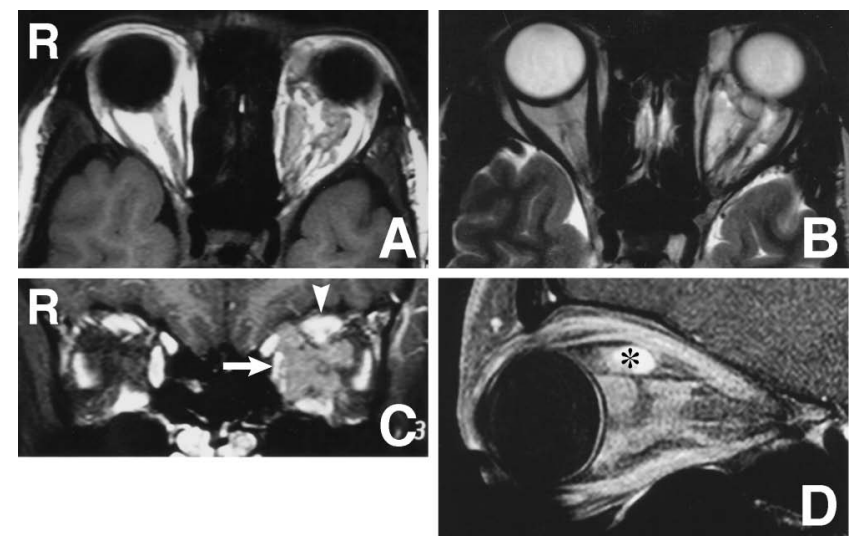

Fig. 2 Axial $\mathrm{T}_{1^{-}}$(A) and $\mathrm{T}_{2}$-weighted (B) magnetic resonance (MR) images showing the lesion as isointense and hyperintense, respectively, compared to the extraocular muscles. Coronal (C) and sagittal (D) $\mathrm{T}_{1}$-weighted MR images with gadolinium revealing absence of enhancement and concomitant vascular lesions. Asterisk: cyst component of the tumor, arrowhead: superior rectus muscle, arrow: medial rectus muscle.

tival chemosis or periorbital ecchymosis, in the affected orbit. These symptoms did not vary with posture, straining, and Valsalva maneuver. Bruit was not audible around the affected orbit. His visual acuity was not reduced compared with the previous examination. The extraocular movements were in-

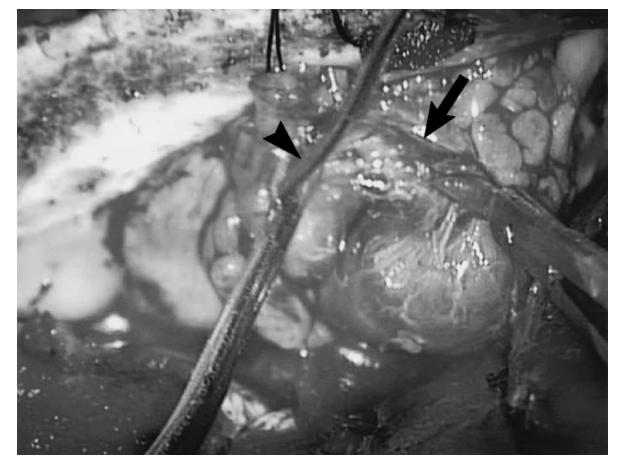

Fig. 3 Intraoperative photograph showing a purplish elastic soft mass, which was exposed as a part of the irregularly-shaped lesion following dissection between the superior (arrowhead) and medial rectus muscles (arrow), buried in the orbital fat.
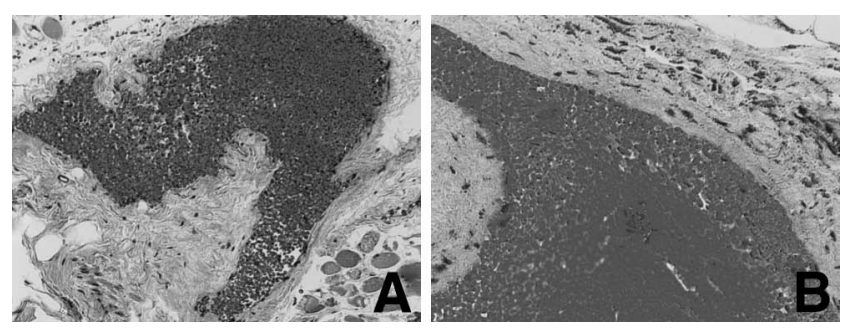

Fig. 4 Photomicrographs showing endotheliallined, thin-walled rounded vessels filled with clot, without smooth muscle or elastic fibers, compatible with orbital cavernous hemangioma. Hematoxylin and eosin stain, original magnification $\mathrm{A}: \times 40, \mathrm{~B}: \times$ 100.

tact with isocoric pupil and preserved light reflex. He was afebrile, and blood examination showed white blood cell count of $5600 / \mu \mathrm{l}$ and C-reactive protein of less than $0.3 \mathrm{mg} / \mathrm{dl}$, in addition to no findings suggestive of coagulopathy.

Computed tomography revealed a homogeneously isodense multiloculated mass, with little discernable cleavage from the medial rectus muscle, mainly located in the medial compartment within the muscle cone of the left orbit, and attached to the lower surface of the optic nerve. No calcification was recognized. The orbital bony structures were intact (Fig. 1). Magnetic resonance imaging demonstrated encasement of the optic nerve by the lesion, which appeared as isointense on $\mathrm{T}_{1}$ - and hyperintense on $\mathrm{T}_{2^{-}}$ weighted images, without enhancement after gadolinium administration. A cyst component was found in the superomedial portion of the lesion. 
Neither vascular malformation nor engorgement of the superior ophthalmic vein was found (Fig. 2).

The patient underwent transcranial surgical exploration. The presurgical diagnosis was dermoid cyst, epidermoid cyst, or thrombosed varix. Superior orbitotomy revealed the intact periorbita underneath. A surgical window was formed by dissecting between the superior and medial rectus muscles, which exposed part of the purplish and elastic soft tumor buried in the orbital fat. The tumor was poorly circumscribed and consisted of a soft part typical of $\mathrm{CH}$, a cystic component including chocolatecolored fluid hematoma, and granulation tissue adhered and intermingling with the perilesional orbital fat (Fig. 3). Circumferential separation from the surrounding musculoadipose tissue was not feasible even with the cryoprobe because of the significant adhesion and the risk of worsening postoperative visual impairment. Consequently subtotal removal was carried out. No vascular lesions were found within the operating field.

Histological examination showed endotheliallined, thin-walled rounded vessels filled with clot, lacking smooth muscle or elastic fibers. The histological diagnosis was $\mathrm{CH}$ (Fig. 4). The postoperative course was uneventful except for transient exacerbation of palpebral ptosis that resolved within 1 month.

\section{Discussion}

Orbital $\mathrm{CH}$ occurs as a well-defined, encapsulated intraconal mass, with sharply demarcated border from the optic nerve, extraocular muscles, and surrounding fatty tissue. Orbital $\mathrm{CH}$ usually appears as homogeneously isointense to muscle on $\mathrm{T}_{1^{-}}$and hyperintense on $\mathrm{T}_{2}$-weighted images, typically with total and homogeneous enhancement after gadolinium administration, but without intralesional calcification which has been identified in $60 \%$ of $\mathrm{CNS} \mathrm{CH}$. Orbital $\mathrm{CH}$ s are firm, whereas intracranial $\mathrm{CHs}$ are bluish, friable lesions. ${ }^{8)}$ All surgical cases of orbital CHs were firm with a hard and compact capsule, and could be totally removed by careful circumferential dissection. ${ }^{1)}$ Histological examination in all cases found endothelial-lined, thin-walled rounded vessels without smooth muscle or elastic fibers, and the collagenous tissue forming the vessel walls was not degenerated, in contrast to the findings for CNS CHs. Present case showed atypical presentation on neuroimaging. An irregular contour without enhancement was atypical for $\mathrm{CH}$, whereas intraoperative findings were consistent with that of $\mathrm{CH}$, although the portion appearing $\mathrm{CH}$ represented only a small part of the orbital pathology. Microscopic findings, on the other hand, were compatible with $\mathrm{CH}$ without coexisting vascular pathologies. We assume that offending lesion might be heterogenous in consistency and include coexisting abnormal venous structures which had thrombosed completely before evaluation by imaging study.

Symptomatic orbital CHs are usually treated by microsurgical total removal with satisfactory longterm outcome. ${ }^{1,3,5,10)}$ In contrast, conservatively managed patients remained stable during a followup period of 3-10 years.7) Present case showed progressive ocular symptoms caused by the orbital lesion and underwent a surgical exploration. To date, three atypical cases of orbital $\mathrm{CH}$ tightly adhered to the optic nerve and retrobulbar tissue have been reported. ${ }^{11}$ Among them two cases were incompletely extirpated with no regrowth for 2 years. However, surgery in one case resulted in damage to the optic nerve caused by pulling and rotating movements during the operation, with complications of low vision and mydriatic pupil.

Scientific discussion of orbital vascular malformations has long been hampered by conflicting definitions and terminology. Orbital vascular anomalies, especially in pediatric populations, tend to occur as combined lesions, so classification has tended to be somewhat controversial. Classification according to the hemodynamic relationships into no flow malformations, venous flow malformations, and arterial flow malformations should reduce the clinical confusion resulting from purely morphologic differentiation. ${ }^{2)}$ This classification may partly explain the heterogeneous clinicopathologic spectrum of orbital $\mathrm{CHs}$ beyond the limitations inherent to the morphological identification. One case of pediatric orbital tumor occurred as a combination of malformed ectatic veins intermingled with dilated lymphatic channels, and focal cavernous and capillary hemangiomatous features. ${ }^{9)}$ No specific disease entities were identified, and consequently the diagnosis was vascular hamartoma.

The diagnosis in the present case was $\mathrm{CH}$, although total tumor resection was not possible because of tight adhesion, so the remaining portion of the irregularly-shaped heterogeneous lesion was not histologically examined. Transconjunctival approach combined with eyebrow incision can provide less invasive access to all orbital quadrants except for lesions with intracranial or intrafacial tumor extension and pathological entities located purely in the orbital apex.1.5) In the present case, preoperative neuroimaging revealed that the lesion involved all orbital quadrants, although mainly the medial compartment, and extended to the orbital apex. In addition, the tumor was irregularly shaped 
and poorly circumscribed from the surrounding neuromuscular structures, which suggested tedious intraoperative dissection and possible significant bleeding. Therefore, we selected the transcranial route which provides a wide operating field and enables safe surgery under the operating microscope. Cryoprobe-assisted surgery is effective and useful for orbital tumor resection, but mainly used by ophthalmologists. Orbital surgery can be performed with minimal bleeding using a cryoprobe, without jeopardizing the capsule of the tumors. ${ }^{4)}$ However, the cryoprobe was ineffective for the hard adhesion in the present case, which may also reflect the peculiar aspects of the present hemangioma not evaluated by presurgical neuroimaging.

Orbital $\mathrm{CH}$ may present with an atypical appearance which confuses the pretreatment diagnosis and makes surgical extirpation more hazardous. Conservative surgery should be indicated for poorly demarcated orbital CHs considering the usual benign clinical course and postoperative sequelae.

\section{References}

1) Cheng JW, Wei RL, Cai JP, Li Y: Transconjunctival orbitotomy for orbital cavernous hemangiomas. Can J Ophthalmol 43: 234-238, 2008

2) Harris GJ: Orbital vascular malformations: a consensus statement on terminology and its clinical implications. Orbital Society. Am J Ophthalmol 127: 453-455, 1999

3) Hejazi N, Hassler W, Offner F, Schuster A: Caver- nous malformations of the orbit: a distinct entity? A review of own experiences. Neurosurg Rev 30: 54-55, 2007

4) Hurwitz JJ, Mishkin SK: The value of cryoprobe-assisted removal of orbital tumors. Ophthalmic Surg 19: 94-97, 1988

5) Koerbel A, Ferreira VR, Kiss A: Combined transconjunctival-eyebrow approach providing minimally invasive access to all orbital quadrants. Technical note. Neurosurg Focus 23(5): E10, 2007

6) Ohtsuka K, Hashimoto M, Suzuki Y: A review of 244 orbital tumors in Japanese patients during a 21-year period: origins and locations. Jpn J Ophthalmol 49: 49-55, 2005

7) Scheuerle AF, Steiner $\mathrm{HH}$, Kolling G, Kunze S, Aschoff A: Treatment and long-term outcome of patients with orbital cavernomas. Am J Ophthalmol 138: 237-244, 2004

8) Thorn-Kany $M$, Arrue $P$, Delisle MB, Lacroix F, Lagarrigue J, Manelfe C: Cavernous hemangiomas of the orbit: MR imaging. J Neuroradiol 26: 79-86, 1999

9) Woo KI, Kim YD: Vascular hamartoma of the orbit. Korean J Ophthalmol 14: 103-106, 2000

10) Yan J, Wu Z: Cavernous hemangioma of the orbit: analysis of 214 cases. Orbit 23: 33-40, 2004

Address reprint requests to: Satoshi Tsutsumi, M.D., Department of Neurological Surgery, Juntendo University Urayasu Hospital, 2-1-1 Tomioka, Urayasu, Chiba 279-0021, Japan. e-mail: shotaro@juntendo-urayasu.jp 